2 Preston FE, Makris M, Triger DR, Underwood JCE. Prevention of hepatitis $C$ virus infection in haemophiliacs. Lancet 1990;336:63.

3 Habibi B, Garretta M. Screening for hepatitis C antibody in plasma for fractionation. Lancet antibody in plasm

4 Cash J. Screening for hepatitis $C$ virus antibody in plasma for fractionation. Lance 1990;335:1216.

\section{Platelet aggregation in Raynaud's} phenomenon

Biondi and Marasini recently reported that patients with Raynaud's phenomenon showed increased platelet aggregation induced by serotonine and adenosine diphosphate (low doses), and normal platelet aggregation induced by adrenalin.'

We also investigated adrenaline $(5 \mu \mathrm{g} / \mathrm{ml})$ induced platelet aggregation in 20 healthy volunteers, 27 patients with primary Raynaud's phenomenon, and 25 patients with obliterative atherosclerosis. We registered the time to the start of aggregation rather than its intensity. The mean (SD) figures were $34 \cdot 2$ (5.57) seconds in Raynaud's phenomenon and $37.8(5.54)$ seconds in obliterative atherosclerosis. The time registered to the start of aggregation was significantly shorter in Raynaud's phenomenon compared with that in normal adults $(46.3(4.37)$ seconds ( $p$ $=0.01)$ and even with that in atherosclerotic patients $(p=0.05)$.

It is interesting to note that both the time to the start of aggregation and its intensity are abnormal in patients with Raynaud's phenomenon. The observation of both variables may be useful in such patients.

GE SLUTSKER GE SLUTSKER
Kuibyshev Medical Institute,
Post Box 703,
Post Office 100,
Kuibyshev (Samara), 443100 Kuibyshev ( Samara), 443100
USSR

1 Biondi L, Marasini B. Abnormal platelet aggregation in patients with Raynaud's phenomenon. J Clin Pathol 1989;42:716-8.

Immature lymphocytes in transient erythroblastopenia of childhood

The report by Foot et al on bone marrow lymphocytes in transient erythroblastopenia of childhood (TEC) ${ }^{1}$ is important because it redirects our attention to the patterns of immature lymphocytes which may be found in children's bone marrows. Such cells were once called haematogones. A recent study by Longacre et al described detailed studies of these cells in 12 children with a variety of malignant and non-malignant disorders, among which were three cases of red cell aplasia. ${ }^{2}$ They showed a complex pattern of phenotypic and morphological appearances of these lymphoid cells. These observations highlight what should now be axiomatic for haematologists: cell marker studies should not be used to make a diagnosis of leukaemia, but, once such a diagnosis has been made by the usual methods, may give an indication of what sort of leukaemia it is.

Foot $e t$ al also wonder why bone marrow lymphocytosis should occur in TEC. Among a range of possibilities is the fact that normal children of this age may have up to, or more than, $40 \%$ lymphocytes in their marrow. Removal of the erythroblast population, say $20 \%$, could result in the lymphocytes reaching $50 \%$ of the total nucleated cell population without any apparent reduction in the cellularity of the sample, and without an absolute increase in the number of lymphocytes. "Lymphocytosis" in the bone marrow is of course relative. Nevertheless, the increased proportion of early lymphoid cells in the mononuclear cell population obtained by density separation does suggest that it may be "a consequence of an outpouring of immature lymphocytes," unless a corresponding decrease in the absolute number of mature lymphocytes has occurred. Perhaps all three of these processes contribute to the increased proportion of immature lymphoid cells in the bone marrow of those with TEC. Royal Vichoematology, Royal Victoria Infirmary, Newcastle upon Tyne
NE1 4 LP.

1 Foot ABM, Potter MN, Ropner JE, Wallington TB, Oakhill A. Transient erythroblastopenia of childhood with CD10, TdT, and cytoplasmic u lymphocyte positivity in bone marrow. J Clin Pathol 1990;43:857-9.

2 Longacre TA, Foucar K, Crago S, et al. Haematogones: a multiparameter analysis of Haematogones: a multiparameter analysis of
bone marrow precursor cells. Blood 1989; 73:543-52.

\section{Immunoalkaline phosphatase technique in renal pathology}

It was a pleasure to read the article by Jackson et al regarding the immunoalkaline phosphatase technique on formalin fixed renal biopsy specimens. We are writing merely to comment on two problems outlined by the authors in their article.

The problem of weak or negative staining encountered in cases of anti-glomerular basement membrane disease (anti-GBM) may result from the fixative used; buffered formalin has a stronger effect on the antigenicity than acid formalin and also requires a greater digestion time to unmask the epitopes. By using formol saline, we have much shorter digestion times in trypsin and the staining of complement is usually stronger. We find $\mathrm{C} 3$ of more diagnostic value than IgG in cases of anti-GBM disease probably because of the lower background staining.

The other problem of spurious staining of plasma in capillary loops can be reduced or even stopped by washing the specimen in physiological saline for around one hour before fixation.

We use immunoperoxidase routinely on renal biopsy specimens as well as immunofluorescence performed in another department. Having read the article by Jackson et al ' we will be assessing the immunoalkaline phosphatase technique.

\section{A AGUIRREBURUALDE A J HOWAT Department of Histopathology Royal Preston Hospital, PO Box 202, Sharoe Green Lane,}

1 Jackson R, Holme ER, Phimister GM, Kennedy A, McLay ALC. Immunoalkaline phosphatase technique applied to paraffin wax embedded tissues in diagnostic renal pathology. J Clin Pathol 1990;43:665-70.

\section{BOOK REVIEWS}

Diagnostic Seminars in Pathology. Vol 1. Ed E Grundmann. (Pp 318; 45 tables; soft cover DM 89.). Gustav Fischer. 1990. ISBN 3437113364

This new series "summarises articles previously published in pathology-research and practice". Volume I contains nine articles on neuroendocrine tumours, and single articles on prostatic carcinoma, cytological diagnosis of lung cancer, myositis, viral encephalitides, storage disorders, electron microscopy of large cell undifferentiated and giant cell tumours, Niemann-Pick diseases, chronic renal failure, and the use of lectins in histopathology. With such diverse subject matter this book may not immediately appeal to pathologists as "an up to date reference source", but I must confess to finding several of the articles most informative and helpful. The chapter on neuroendocrine tumours of the gastrointestinal tract is a gem; if you are not quite clear about enterochromaffin-like (ECL) hyperplasias and neoplasias of the stomach in relation to various stimuli then this chapter will sort things out. It concludes with a most useful and erudite discussion of the terminology of gut neuroendocrine tumours and the use of the term "carcinoid" The chapter on phaeochromocytomas and paragangliomas begins with very clear definitions of these tumours. The chapter on thymic neuroendocrine neoplasms is especially useful in its discussion of the differential diagnosis of such tumours. This is certainly a book that candidates for final MRCPath would be well advised to dip into.

DA LEVISON

Macro Techniques in Diagnostic Histopathology. DG Lowe, IM Jeffrey. (Pp 144; $£ 40$.) Wolfe. 1990. ISBN 0723409455.

Many histopathologists learn to deal with specimens in an apprenticeship of varying length, collecting tips haphazardly from older colleagues whose skill was similarly acquired. Even in maturity our reports may not always make it clear to clinicians or reviewing pathologists precisely what we found.

Following the advice of this attractive, highly practical guide to specimen examination, description and block selection, should result in consistent high quality macroscopical reports and proper blocks.

Fourteen short chapters on different systems are written in an easy, carefully edited style with excellent closely matched photographs, tables, and diagrams. The 100 specimens illustrated well represent the daily work of the average histopathology laboratory. Procedures suggested are consistent and reasonable, although some migh baulk at the number of blocks advocated. Clarification of why certain blocks are taken might have been desirable. None the less I wish I had this volume when I started.

AM MACKAY

Progress in Reproductive and Urinary Tract Pathology. Vol 1. Ed I Damjanov, AH Cohen, SE Mills, RH Young. (Pp 217 49.) John Wiley. 1990. ISBN 0938607138

The editors have embarked on a new series of books containing "review articles ... . written 
by pathologists for pathologists", and volume 1 compares favourably with the Recent Advances in Pathology series. The articles cover aspects of gynaecological, urological, and nephrological pathology.

Much of this volume describes cancers with low malignant potential, and the term "borderline" in ovarian tumours is used not only for serous and mucinous tumours, but also endometrioid, clear cell, Brenner and mixed Mullerian tumours. The diagnosis of persistent and proliferative gestational trophoblastic disease is clearly described.

There are chapters on early prostatic malignancy, and the lack of editorial censorship is shown by one author warning of the risks of treating incidental prostatic carcinoma, while the subsequent chapter advocates radical prostatectomy for similar lesions. The book also describes papillary prostatic urethral lesions and malignant testicular stroma tumours, and there are chapters on the multiple causes of crescentic glomerulonephritis and fibrillary glomerulonephritis.

This is a very valuable collection of articles, and reasonably priced. If subsequent volumes are of a similar standard Progress in Reproductive and Urinary Tract Pathology will be a very welcome series of publications.

KM GRIGOR

Cyclosporin. Mode of Action and Clinical Application. Ed AW Thomson. (Pp 372; £50.) Kluwer Academic Publishers. 1990. ISBN 0 7462-0124-9.

It is trite to note that the use of cyclosporin has greatly enlarged the clinical possibilities of transplantation, and it is a truism to state that trying to understand the mechanism of its actions has pained immunologists by showing up a huge area of their ignorance. The understanding of molecular and cellular signal transduction and effector activation are being applied to cyclosporin and its new rival FK506, but there is still much to learn-and much to be learnt from studies of the pharmacology of cyclosporin.

Dr Thomson, whose own work has combined functional and morphological investigations in this field, has edited an attractive and useful synoptic account of our general understanding up to early 1989 . There are 15 chapters by active scientists and clinicians from Australia, Britain, France, Switzerland and the USA. They review in varying detail the effects of cyclosporin A on mechanisms of cellullar and humoral immune response initiation and amplification $(20 \%$ of the book), and its therapeutic potential or proven value in human diseases of the bone marrow, eye, diabetes, skin and autoimmune disorders $(40 \%)$. The remaining third covers pharmacokinetics, drug metabolism-harmful effects in clinical practice and pathological changes in experimental models.

Each chapter contains a tidy statement of current knowledge, plentiful illustrations, and a good supply of references. Most can only report phenomena, as our understanding is deficient, but others do discuss likely mechanisms involving binding to a specific cytoplasmic protein and downstream consequences on interleukin 2-mediated cell activation and proliferation.

To the basic scientist the book offers a useful but inevitably slightly dated review. For the applied researcher and clinician it provides valuable guidance on the possibilities and problems of treatment. For a compound with such a narrow therapeutic range it is disappointing that almost no author describes the detail of any dosing regimen used-the drug is just administered! Apart from that lapse, $\mathrm{Dr}$ Thomson has provided a book of great use to immunologists and pathologists.

AD DAYAN

Management of Orbital and Ocular Adnexal Tumours and Inflammations. Ed JA Mauriello, JC Flanagan. (Pp 285; Hardcover DM 280.00.) Springer. 1990 ISBN 3540511555 .

This is a profoundly disappointing book which is a great pity because it contains some very good things. These include excellent photomicrographs, clinical illustrations, and gross pathological photographs, many of rare entities, culled from AFIP alumni and from Dr Flanagan's extensive practice. There are also a large number of well organised tables throughout the book. These good points only serve to highlight the deficiencies and chao of the text. Although designed to be dipped into, rather than read as a whole, the organisation of the text constantly flits from one topic to another and frequently places a whole team of horses before the cart. I asked two "busy ophthalmologists", at whom the book is aimed, to look at it; both found the format and indexing irritating and elusive. From the pathologist's standpoint it is unsatisfactory to have paragraph headings of conditions that are not synonymous and to describe only the first, an example being: angiolymphoid hyperplasia (Kimura's disease, eosinophilic granuloma, eosinophilic folliculosis) when only Kimura's disease is described. Pick ou the plums and you will enjoy it. Read it all and you risk indigestion.

ACE MCCARTNEY

Pharmacology-Drug Actions and Reactions. Ruth R Levine. (Pp 576; sof cover $£ 24.95)$ Churchill Livingstone. 1990 ISBN 0-316-52217-1

This book is a useful introduction to the concepts of pharmacology and toxicology. Many medical graduates will have covered most of the ideas presented in their undergraduate years. The clinical scientist or MLSO rotating through his or her department's drug analysis section for the first time, however, will find it invaluable. Indeed, the hardest part of writing this review has been prising the book loose from the toxicology section, where it has rapidly become a fixture on the bookshelf alongside Clark, Goodman, and Gilman, the British National Formularly, and the Data Sheet Compendium. Strongly recommended: a definite "best buy". ARW FORREST

\section{NOTICES}

\section{ACP Locum Bureau}

The Association of Clinical Pathologists runs a locum bureau for consultant pathologists.

Applicants with the MRCPath who Aould like to do locums and anyone requiring a locum should contact The General Secretary, School of Biological Sciences, Falmer, Brighton, BN1 9QC Tel and Fax: 0273678435.

\section{Lung Pathology}

London, 10-12 June 1991

A comprehensive course of lectures, hands-on microscopy sessions, and a slide seminar will be held at the Brompton Hospital. The programme will include J Wigglesworth on perinatal disease, $M$ Dunnill on defence mechanisms and fibrosis, A Gibbs on pneumoconiosis, $\mathrm{C}$ Wagenvoort on hypertension and a variety of internal speakers on airway disease, infections, interstitial disease, angiitis and tumours.

\section{Fee $£ 150$ (or US\$290).}

Applications to Professor B Corrin, Histopathology, Brompton Hospital, London SW3 6NP.

\section{Centre for Health Planning and Management \\ Diploma in Management (Diagnostic Services)}

Applications are invited for places on this part-time diploma, beginning in October 1991. It is aimed at heads of department and potential heads in Pathology.

The Diploma in Management covers applied management principles, health policy, management of human resources and operations management. It aims to provide the candidate with a sound backing in both scientific and behaviourial aspects of management, and the curriculum relates to the NHS of the 1990s and beyond. Potential applicants wishing to discuss the programme further should contact either Professor Roger Dyson or Dr Calum Paton on 0782 621111 (ext 3646).

Further details and full application materials are available from: Tanya Matthews, Centre for Health Planning and Management, Suite 2.1, Science Park, University of Keele, Staffordshire, ST5 5SP

\section{Corrections}

An error appeared in the bottom line of the first column of the table in the letter, "Are calculated globulin measurements useful in screening for paraproteinaemia?" ( $J$ Clin Pathol 1990;43:694). The correct line should have read:

Specificity $=\mathrm{TN} / \mathrm{FP}+\mathrm{TN} \times 100=$ $96 \cdot 7 \%$.

Two authors names were omitted from a letter to the Editor, "Breast carcinoma cellularity and its relation to oestrogen receptor content." (J Clin Pathol 1989;42:1166-8). The names of P Coy of the Victoria Cancer Clinic, The Cancer Control Agency of British Columbia, and C Fletcher of the Special Development Laboratory, Greater Victoria Hospital Society, should have been included. 